\title{
Particle heating in a thermal plasma
}

\author{
Xi Chen \\ Department of Engineering Mechanics, Tsinghua University, Beijing 100084 , \\ People's Republic of China
}

\begin{abstract}
Eome aspects of the recent progress in plasma-particle heat $\overline{\text { transfer }}$ study are reviewed with emphasis on heat transfer mechanisms, effects of the Knudsen number on the heat transfer and some other complicated factors related to the plasma-particle heat transfer. Although conduction/convection is the main heat transfer mechanism, sometimes one has to consider many other mechanisms and complicated factors such as radiation, ion-electron recombination at the particle surface, the knudsen effect, particle evaporation, plasma-particle interactions, etc. Heat transfer expressions for different heat transfer regimes from continuum to FMF are proposed and compared with corresponding experimental data. It is indicated that more careful experimental studies are needed to clarify the effects of non-LTE and other complicated factors and to check the available heat transfer correlations for the case of small particle sizes.
\end{abstract}

\section{INTRODUCTION}

Knowledge concerning how to estimate and suitably control particle trajectories and particle heating histories is a key requirement raised by many applications of the thermal plasma processing of particulate matter. Several examjles of the actual applications are plasma spraying [ref. 1], plasma decomposition and synchesis [ref. 2], ICP acomic spectroscopy [ref. 3], etc.

Numerical modeling has been widely employed to quanticatively study various different processes, including particle movement and heating involved in the thermal plasma processing. It has been recognized that modeling is a powerful tool to optimize process parameters of an estajlished plasma processing equipment and to predict the performance of a planning plasma processing route. It is especially true if some parallel experimental investigations are conducted to check the modeling predictions and/or to provide supporting data to the modeling work.

There exist two different approaches to model numerically the thermal plasma processing of pariculate matter: the quasi-one-dimensional particle loading plasma two-phase flow approach [refs. 4-6] and the two-dimensional plasma flow - particle trajectory approach [refs. $7-16]$. However, both approaches all require information concerning heat transfer and drag between plasma flow and injected particles. Usually, particle trajectories and particle heating histories are calculated by solving equations with appropriate initial and boundary conditions for particle movement and for unsteady heat conduction within the particle. It is, perhaps, the reason why many researchers in many countries working in the field of thermal plasma processing show their constant interest in the study of che plasmaparticle heat transfer [refs. $1-36$.

Since Dr. Waldie's excellent review paper was published 15 years ago [ref. 37], a great progress has been achieved in the quantitative calculation of plasma-particle heat transfer under various different plasma/particle conditions and with accounting for many complicaced factors affecting particle heat transfer and drag. Some recent progress has been reviewed in Refs. [20,38-41, $1 \& 3]$. The present paper also intends to review some aspects of the recent progress. Refs. [38 - 40] discussed in some detail various different factors which may affect the motion of a particle in a thermal plasma flow, including strongly varying plasma properties, non-continum effect, the Basset history term, thermophoresis, turbulent dispersion, etc. The relative importance of these factors was assessed for modeling of particle movernent in the the rmal plasma processing of particulate matter. It was also expounded in refs. [38 - 41] how to calculate particle trajectories and heating histories with taking many complicated factors inco account. Hence, the present paper will concentrace main attention on the basic processes of plasma-particle heat transfer. 


\section{HEAT TRANSFER MECHANISMS}

Since smaller particle sizes (e. g. tens of microns are typical for plasma spraying, while microns or even submicrons are encountered in the ICP atomic spectroscopy) and greater mean free path lengths (microns under atmospherical pressure and increase with the reduction of the gas pressure) are involved in many applications, various different heat transfer regimes can be met depending on the Knudsen number ( $\mathrm{Kn}$, defined as the ratio of the mean free path length of gas particles to particle diameter). This problem will be discussed in some detail in the next section of the present paper.

Due to smallness of the particle size and the corresponding Grashof number, natural convection is in general negligible in the plasma-particle heat transfer calculation. In addition, Reynolds numbers are also comparatively small, so that heat conduction usually can not be ignored in comparison with the forced-convection effect even for higher oncoming plasma flow velocity and/or greater particle sizes.

Since the shielding parameter is usually much less than 1.0 and the plasma can be treated as optically thin, there exists radiative energy exchange between the particle in study and the plasma reactor wall or cold surroundings "seen" by the particle. Radiation from the particle surface to cold surroundings or reactor wall is readily calculated, and it is negligible only as low particle surface temperature, small particle sizes and/or high enthalpy plasmas are concerned [refs. $24 \& 26$ ]. The shielding parameter is defined as the ratio of the total cross section area of the other particles "seen" by the particle in study to the outer surface area of the bulk plasma; and this parameter can be expressed as

$$
\frac{\pi r_{w}^{2} n \pi R^{2} H}{2 \pi R H+2 \pi R^{2}}=\frac{3}{8}\left(\dot{m}_{w} / \dot{m}_{p}\right)\left(\rho_{p} / \rho_{w}\right)\left(\frac{R}{r_{w}}\right)\left[R H /\left(R H+R^{2}\right)\right]
$$

where $R$ and $H$ are radius and height of the hot plasma region, $\left(\dot{m}_{w} / \dot{m}_{p}\right)$ is the particle/plasma mass-flow-rate ratio, $\left(\rho_{p} / \rho_{w}\right)$ is the plasma/particle-material density ratio, $r_{w}$ and $n_{w}$ are the average radius and number density of injected particles in the particle loading plasma two-phase flow, respectively. For a dense particle loading plasma two-phase flow, radiation from other particles to the particle in study may be important as particle surface temperatures are high, but so far this problem is not well studied.

Radiation from plasma flow to the particle is often ignored in the plasma-particle heat transfer calculation, but it is not always justified. For a particle with radius $r_{w}$ immersed in a thermal plasma flow with outer radius $R$, the radiative heat flux from the plasma to the particle can be estimated as

$$
q_{r}=\frac{1}{\left(4 \pi r_{W}^{2}\right)} \int_{r_{w}}^{R} \varepsilon U_{r}\left(4 \pi_{r}^{2}\right)\left(r_{w} / r\right)^{2} d r=\varepsilon U_{r}\left(R-r_{w}\right)
$$

The conductive heat flux is [refs. $23 \& 26$ ]

$$
\mathrm{q}_{\mathrm{c}}=\left(\mathrm{s}_{\mathrm{p}}-\mathrm{S}_{\mathrm{w}}\right) / \mathrm{r}_{\mathrm{w}}
$$

Bence the radiative/conductive heat flux ratio is obtained

$$
\left(q_{r} / q_{c}\right)=\varepsilon r_{w} U_{r}\left(R-r_{w}\right) /\left(s_{p}-s_{w}\right)
$$

Where $U_{\text {. }}$ and $S$ are cemperature-dependent radiation power per unit volume and the heatconduction-potential [ref, 23]; $\varepsilon$ is the particle surface emissivity; subscripts $p$ and $w$ express bulk plasma and particle wall, respectively. It is obvious that the heat flux ratio $\left(q_{r} / q_{c}\right)$ is directly proportional to the particle radius and the plasma dimension $R$. Supposing $r_{w}=50$ microns and $R=1 \mathrm{~cm}$, the calculated results of $\left(q_{r} / q\right)$ as function of plasma temperature is shown in $F i g .1$. The radiation heat transfer ma $f_{e}$ as great as $18 \%$ of the conductive heat flux for this sample case and for argon plasma, as seen from Fig. 1. Radiation from plasma flow to a particle may be important as big particle sizes, large plasma dimensions and high plasma temperatures are concernec. It can be ignored for smaller particles with racii of a few microns.

The importance of patticle evaporation (if evaporating particles are concerned) and the Knucisen effect on plasma-particle heat transfer has been recognized. For an evaporating or sublimating particle, the outflow gas from the particle would reduce significantly heai flux to the particle in comparison with the case of non-evaporating particle [ref. 23]. The Knucisen or rarefacion effect is an important mechanisin causing pronounced reduction of the plasna-paricicle heat flux as small particle sizes and/or low gas pressures are involved [ref. 25]. Heat transfer is enhanced for the case with convection. If convection effect is included in the analysis, calculation concerning the Knucsen effect or evaporation effect on plasma-particle heat transfer would be much complicated. Fortunacely, it has been shown 


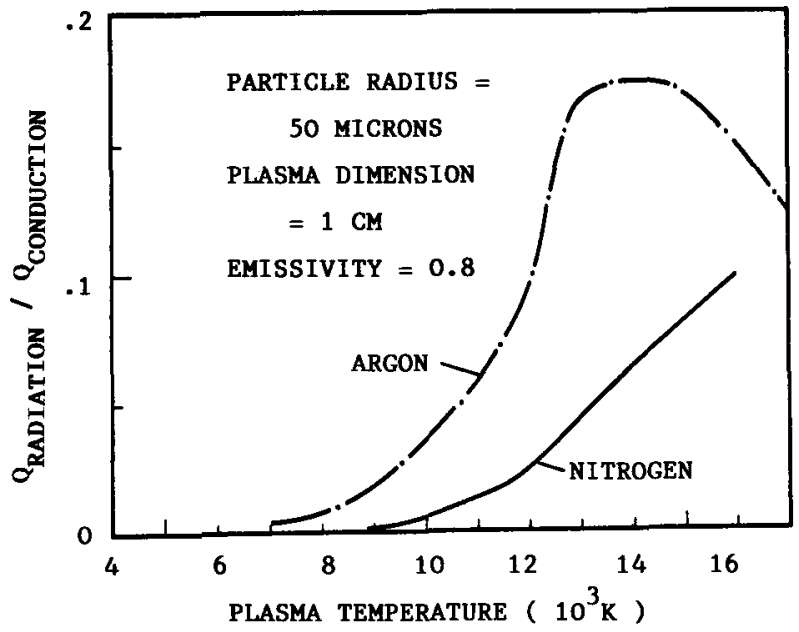

Fig. 1. Variation with plasma temperature of the radiation /conduction heat flux racio for argon and nitrogen plasmas.

that the analytical results obtainec for the case with convection concerning the heat flux ratio with to without accounting for the evaporation effect or the Nnucsen effect rexain almost the same as those for the case without convection under typical conditions for thermal plasma processing [refs. 23 \& 27]. These findings obtained based on computational scudies would be helpful to simplify significantly the calculation of plasma-paricle heat transfer including these complicated factors in the modeling of thermal plasma processing.

Ref. [28] studied the effect on heat transfer due to vapor contamination from evaporating particles. The heat flux from the concaminated plasma to the particle in study was shown to be different appreciably from that for the case of "pure" plasma. Accurate calculation including this effect requires using temperature-dependent plasma properties for various different plasma/vapor compositions.

Under dense particle loading conditions, particle heating and accelaration can appreciably affect local plasma temperature and velocity, and thus affect plasma-particle heat transfer itself [ref. 16]. Ref. [6] in which a few typing mistakes appeared but did not affect the resulis) showed that the critical value of the local particle/plasma mass-flow-rate ratio beyond which particle-plasma interaction effects become important depends on the desired temperature level of the heated particles, on the plasma temperature and on the type of particle material.

Electron emission from a metallic particle represents a heat loss mechanism:

$$
q_{\text {enis }}=j_{e}(w / e)
$$

where $j$, e and $W$ are the emission current density, elementary charge quantity and the work function of the particle material. As a particle with high surface temperature moves into a colc surroundings, the electron emission may be an important mechanism of heat loss [ref. 42]

Charging of particle injected into a thermal plasma flow is very fast [ref. 34]. Relaxation time of the thermal boundary layer around a particle is also very short in comparison with the particle heating time [ref. 26]. Hence, the heat transfer process from plasma to a particle can be treated as quasi-steady for all the particle heating history.

Ion-electron recombination at the particle surface contributes about $10 \%$ or more to the total heat flux [refs. $43 \& 44]$. As a biased voltage is applied between an immersed body and the plasma, electric current can be drawn [refs. $43 \& 44$ ]. These facts imply that some non-LTE features exist within the thermal boundary layer. Although the conductive heat flux can be calculated for a LIE or a frozen boundary layer if corresponding plasma properties are available, the study of plasma-particle heat transfer under more general conditions other than LTE or frozen ones requires using a numerical method and is not well worked out so far.

The electron temperature may be different from the atom and ion cemperature in the thermal boundary layer around the particle, especially as a flowing plasma flow is concerned. The two-temperature situation has been considered in the heat cransfer analysis for the extreme case of free-molecule flow (FMF) regime under plasma conditions [refs. 34 \& 35], but not been well studied for the continuum regime although it is expected that the results for the two- 
temperature case would be different from those for the LTE case. A helpful discussion was given in ref. [45] concerning the heat transfer mechanisms near a catalytic or noncatalytic wall. In this way those authors explained the puzzling phenomenon observed in the temperature measurement of a rod wall during its plasma sintering process.

\section{HEAT TRANSFER FOR DIFFERENT REGIMES}

\section{Continuum regime}

An expression for heat transfer to a spherical particle without evaporation immersed in a continuum thermal plasma flow is of fundamental importance for the modeling work of many applications such as plasma spraying, plasma spheroidizing,etc. It is because that based on such an expression, some other complicated factors can be considered by introducing corresponding correction coefficients, such as evaporation [ref. 23], rarefaction effect under smali Knudsen numbers [refs. 25 \& 27], non-spherical particle shape, vapor contamination [ref. 28], etc. This expression is needed even for the cases with moderate Knudsen numbers if an interpolation formula is employed to calculate the plasma-particle heat iransfer within the transition regime [ref. 14].

The well-known Ranz-irarshall formula and its various differenc modified forms for thermal plasma condicions are widely employed in the modeling of thermal plasma processing:

$$
\begin{aligned}
& \text { [refs. } 4 \& 5] \quad \mathrm{Nu}_{\mathrm{f}}=\mathrm{qd} /\left[\left(\mathrm{T}_{\mathrm{p}}-\mathrm{T}_{\mathrm{W}}\right) \mathrm{k}_{\mathrm{f}}\right]=2+0.6 \mathrm{Re}_{\mathrm{f}}^{0.5} \mathrm{pr}_{\mathrm{f}}^{1 / 3} \\
& {[\text { refs. } 9 \& 14] \quad N u_{\mathrm{f}}=\left[2+0.6 R \mathrm{e}_{\mathrm{f}}^{0.5} \mathrm{Pr}_{\mathrm{f}}^{1 / 3}\right]\left[\left(\boldsymbol{\rho}_{\mathrm{p}} \mu_{\mathrm{P}}\right) /\left(\boldsymbol{\rho}_{\mathrm{W}} \mu_{\mathrm{W}}\right)\right]^{0.6}} \\
& {[\text { refs. } 10 \& 13] \quad N u_{f}=\left[2+0.6 R e_{f}^{0.5} \operatorname{Pr}_{f}^{1 / 3}\right]\left[\left(\rho_{\mathrm{p}} \mu_{\mathrm{p}}\right) /\left(\rho_{\mathrm{w}} \mu_{\mathrm{W}}\right)\right]^{0.6}\left(\mathrm{CP}_{\mathrm{p}} / \mathrm{Cp}_{\mathrm{w}}\right)^{0.38}} \\
& {\left[\text { refs. 7, 8, 15] Nu } u_{f}=\left[2+0.6 R e_{f}^{0.5} \operatorname{Pr}_{f}^{1 / 3}\right]\left[\left(\rho_{p} \mu_{f}\right) /\left(\rho_{f} \mu_{p}\right)\right]^{0.15}\right.} \\
& \text { [refs. } 19 \& 20] \quad N u *=q d p_{w} /\left[k_{p}\left(h_{p}-h_{w}\right)\right]=2 k_{w} / k_{p}+0.5 \operatorname{Re}_{p}^{0.5} \operatorname{Pr}_{p}^{0.4}\left[\left(\rho_{p} \mu_{p}\right) /\left(\boldsymbol{p}_{w} \mu_{w}\right)\right]^{0.2} \\
& \text { [ref. 21] } \quad \mathrm{Nu}_{\mathrm{w}}=2 \mathrm{f}_{\mathrm{o}}+0.473 \mathrm{Pr}_{\mathrm{w}}^{\mathrm{m}} \mathrm{Re}_{0.19} 0.552 \\
& \text { in which } \mathrm{f}_{0}=\left[1-\left(\mathrm{T}_{\mathrm{w}} / \mathrm{T}_{\mathrm{p}}\right)^{1+\mathrm{x}}\right] /\left\{(1-\mathrm{x})\left[1-\left(\mathrm{T}_{\mathrm{w}} / \mathrm{T}_{\mathrm{p}}\right)\right]\left(\mathrm{T}_{\mathrm{w}} / \mathrm{T}_{\mathrm{p}}\right)^{\mathrm{x}}\right\} \\
& \mathrm{m}=0.78 \operatorname{Re}_{0.19}-0.145 \text { and } \mathrm{x}=0.8 \\
& \text { [ref. 11] } \quad \mathrm{Hu} \mathrm{S}_{\mathrm{S}}=\mathrm{qd} /\left(\mathrm{S}_{\mathrm{p}}-\mathrm{S}_{\mathrm{w}}\right)=2+0.514 \mathrm{Re}_{\mathrm{av}}^{0.5} \\
& \text { [refs. } 29 \& 33] \quad \mathrm{Nu}_{\mathrm{s}}=2\left\{1+0.63 R_{\mathrm{p}} \operatorname{Pr}_{\mathrm{p}}^{0.8}\left(\operatorname{Pr}_{\mathrm{v}} / \operatorname{Pr}_{\mathrm{p}}\right)^{0.42}\left[\left(\rho_{\mathrm{p}} \mu_{\mathrm{v}}\right) /\left(\rho_{\mathrm{v}} \mu_{\mathrm{w}}\right)\right]^{0.52 c^{2}}\right\}^{0.5} \\
& \text { in which } c=\left[1-\left(h_{\mathrm{w}} / \mathrm{h}_{\mathrm{p}} ;{ }^{1.14}\right] /\left[1-\left(\mathrm{h}_{\mathrm{w}} / \mathrm{h}_{\mathrm{p}}\right)^{2}\right]\right.
\end{aligned}
$$

where Nu, Re and $P r$ are Nusselt number, Reynolds number and Prandil number; $\rho, \mu, k, C_{p}, s$, $\mathrm{h}$ and $\mathrm{T}$ are plasma density, viscosicy, thermal conductivicy, specific heat at constant pressure, hear-conduction-potential, specific enthalpy and temperature; $q$ and d are specific heat flux and particle diameter: subscipts $w_{y} p$ and $f$ denoce jarticle surface, plasma onconing flow and the $\mathrm{film}$ iemperature $\left[\mathrm{T}_{\mathrm{f}}=\left(\mathrm{T}_{\mathrm{p}}+\mathrm{T}_{\mathrm{w}}\right) / 2\right]$, respectively. 0.19 expresses using $\left[\mathrm{T}_{\mathrm{w}}+\right.$ $\left.0.19\left(\mathrm{~T}_{2}-\mathrm{T}_{\mathrm{w}}\right)\right]$ as the reference temperature; and "av" means using the properties averaged integially from $T_{w}$ to $T_{p}$.

Since heat conduction term can not be neglected in comparison with the convection term, we do not include any heat transfer expressions without the conduction term although they may be applicable to big spheres.

Because exact soluition is available for the case of pure conduction iRe $=0$ ) [refs. 23 \& 26] and the heat conduction represents important fraction in the plasma-particle cotal heat flux even for the cases with forced-convection, we used the exact solution to check the apjicability of the available expressions for plasma-particle heat transfer [refs. 29 \& 33 ]. Sone calculaced resules are shown in Fig. 2 for argon plasma and pure conduction. It is seen from Fig. 2 that as argon plasma cemperature is less than 7000\%, Eq. (1) can precict correct heat flux due to the negligible gas ionization cegree, but iqs. (2) - (6) assume some errors alchough they were reconmended as the improved forms of the original ranz-iraishall formula,

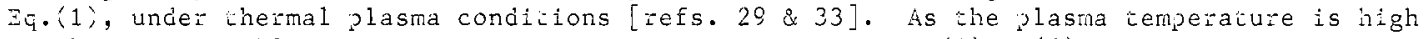
so that appreciade gas ionization appears, none among Eqs. (1) - (5) can be consicered to be sacisfactory. As long as correc values of the cemperature-ciependent chernal conductivity are used, Zis. (7) and (8) can always give correct conductive heat flux since they use an 


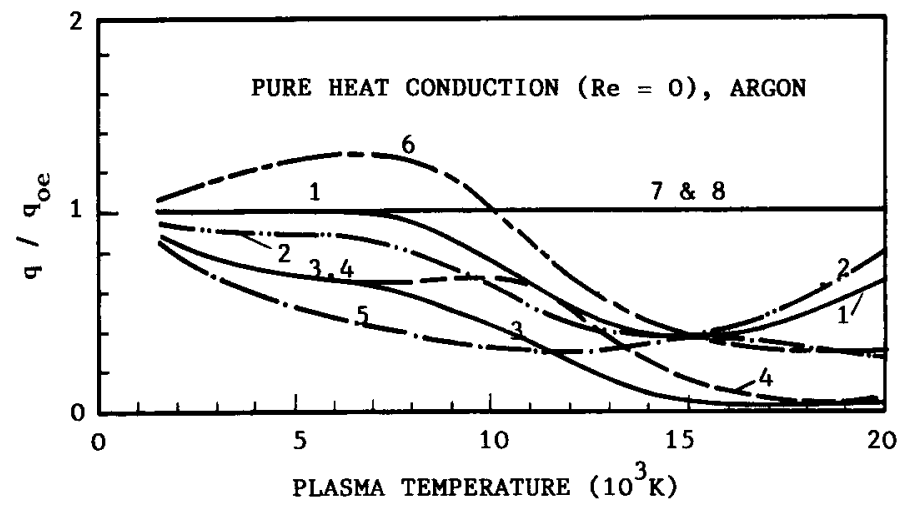

Fig. 2. Comparison of the predicted heat flux by Eqs. (1) - (8) with the exact solution for the case of pure heat conduction and argon plasma $\mathrm{q}_{\mathrm{oe}}=\left(\mathrm{S}_{\mathrm{p}}-\mathrm{S}_{\mathrm{w}}\right) / \mathrm{r}_{\mathrm{w}}, \mathrm{T}_{\mathrm{w}}=$ $1000 \mathrm{~K})$.

appropriate definition of the lusselt number based on the exact solution $q_{o e}=\left(S_{p}-S_{w}\right) / r_{w}$ [refs. 23 \& 26].

Similar calculated results were also obtained for the nitrogen and argon-hydrogen (mole ratio $=1: 4)$ plasmas $[$ refs. $29 \& 33]$.

For the case with the forced-convection effect typical for the thermal plasma processing, comparative study in refs. [29 \& 33] shows that only Eq. (8) can predict heat fluxes in consistence with corresponding computational data for both argon and nitrogen plasmas and for various different plasma/particle parameters (plasma temperature up to $16000 \mathrm{~K}$, relative velocity up to several hundreds $\mathrm{m} / \mathrm{s}$, particle radius from microns to $100 \mathrm{microns}$ ). The computational heat fluxes were obtained by solving the simultaneous governing equations with actual plasma properties as described in refs. [23 \& 27]. Comparisons of the computational heat fluxes with the predictions were given in refs. $[29 \& 33]$ by figures which are not replotted here.

The drawbacks of $\mathrm{Eq}$. (7) are that it does not include the Prandtl number which may vary with plasma temperature appreciably and it also based on a simple summation of the pure conduction term ( $\mathrm{Nu}=2$ ) and the convection term. Based on a power-law summation of the exact solution for the case of pure heat conduction $(\mathrm{Nu}=2$ ) and the corrected simplified analytical result for the case with high Reynolds numbers, the modified heat flux expression, Eq. (8), was proposed based on a combined analytical/computational study [ref. 29]. This modified heat flux expression agrees well with the exact solution for the case of pure heat conduction, and predictions agree with computational data for all the cases studied in refs. [29 \& 33]. Additional comparisons of the predicted heat fluxes by Eqs. (1) - (8) with experimental data recently published [ref. 36] are given in Table 1 and Table 2. Examination of the data shows that Eq. (8) Eits the experimencal data [ref. 36] well for both air and argon plasmas, although other expressions may be applicable to air plasma or to argon plasma.

TABLE 1. Comparison of predicted heat fluxes by Eqs. (1) - (8) with experimental data [ref. 36$]$ for air plasma $(d=6 \mathrm{~mm})$

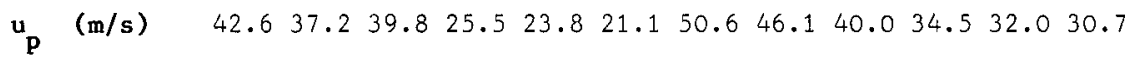

$T_{p} \quad$ (K) 700069006800670066006500720072007100700069006800

$q_{\exp }\left(w / \mathrm{mm}^{2}\right) \quad 8.03 \quad 6.88 \quad 6.65 \quad 5.88 \quad 5.25 \quad 5.02 \quad 9.04 \quad 8.11 \quad 7.25 \quad 6.37 \quad 6.63 \quad 6.30$

Eq.(1) $\quad 4.94 \quad 4.61 \quad 4.65 \quad 3.86 \quad 3.69 \quad 3.47 \quad 5.65 \quad 5.45 \quad 4.99 \quad 4.56 \quad 4.35 \quad 4.21$

$\begin{array}{lllllllllllll}\text { Eq. (2) } & 3.05 & 2.89 & 2.95 & 2.47 & 2.39 & 2.27 & 3.43 & 3.32 & 3.06 & 2.81 & 2.72 & 2.67\end{array}$

$\begin{array}{llllllllllllll}\text { Eq.(3) } \quad 7.66 & 7.13 & 7.17 & 5.91 & 5.62 & 5.25 & 8.42 & 8.11 & 7.58 & 7.06 & 6.73 & 6.49\end{array}$

$\begin{array}{lllllllllllll}\text { Eq. (4) } & 3.94 & 3.69 & 3.73 & 3.11 & 2.98 & 2.82 & 4.48 & 4.32 & 3.97 & 3.63 & 3.98 & 3.38\end{array}$

$\begin{array}{llllllllllllll}q_{t} & \text { Eq.(5) } & 30.5 & 26.9 & 26.0 & 19.5 & 17.5 & 15.3 & 32.4 & 30.9 & 29.3 & 27.5 & 24.9 & 22.9\end{array}$

$\begin{array}{lllllllllllll}\text { Eq. (6) } & 1.10 & 1.03 & 1.04 & 0.86 & 0.82 & 0.78 & 1.21 & 1.17 & 1.09 & 1.01 & 0.97 & 0.94\end{array}$

$\begin{array}{lllllllllllll}\text { Eq.(7) } \quad 9.86 & 17.6 & 17.1 & 13.3 & 12.0 & 10.5 & 5.40 & 5.26 & 6.30 & 9.05 & 16.5 & 15.3\end{array}$

$\begin{array}{lllllllllllll}\text { Eq.(8) } \quad 7.61 & 7.01 & 7.07 & 5.60 & 5.26 & 4.84 & 8.72 & 8.34 & 7.61 & 6.90 & 6.54 & 6.27\end{array}$ 
TABLE 2. Comparison of predicted heat fluxes by Eqs. (1) - (8) with experimental data [ref. 36] for argon plasma (sphere diameter $d=8 \mathrm{~mm}$ )

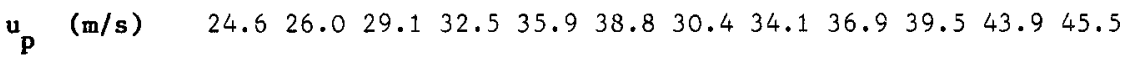

$T_{p} \quad(K) \quad 860086008700870088008900880089009000910092009200$

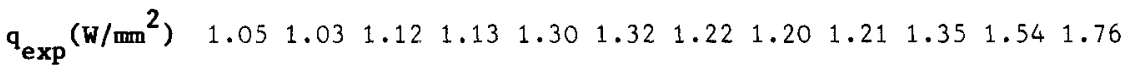

$\begin{array}{llllllllllllll}\text { Eq.(1) } & 1.00 & 1.00 & 1.08 & 1.12 & 1.18 & 1.24 & 1.11 & 1.18 & 1.22 & 1.28 & 1.35 & 1.37\end{array}$

Eq.(2) $\quad \begin{array}{llllllllllllll}0.62 & 0.63 & 0.66 & 0.69 & 0.73 & 0.76 & 0.58 & 0.72 & 0.74 & 0.78 & 0.82 & 0.83\end{array}$

$\begin{array}{llllllllllllll}\text { Eq.(3) } & 0.71 & 0.73 & 0.78 & 0.81 & 0.86 & 0.91 & 0.81 & 0.87 & 0.91 & 0.97 & 1.04 & 1.06\end{array}$

$\begin{array}{lllllllllllll}\text { Eq.(4) } & 0.86 & 0.88 & 0.93 & 0.97 & 1.02 & 1.06 & 0.96 & 1.01 & 1.05 & 1.10 & 1.16 & 1.18\end{array}$

$\begin{array}{llllllllllllll}q_{t} & \text { Eq. (5) } & 0.88 & 0.90 & 0.99 & 1.04 & 1.14 & 1.24 & 1.06 & 1.17 & 1.26 & 1.39 & 1.54 & 1.56\end{array}$

$\begin{array}{llllllllllllll}\text { Eq.(6) } & 0.88 & 0.90 & 0.95 & 0.99 & 1.03 & 1.07 & 0.97 & 1.03 & 1.06 & 1.11 & 1.16 & 1.17\end{array}$

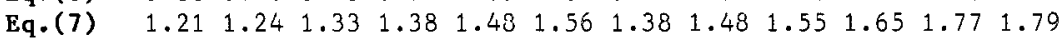

Eq.(8) $\quad 0.98 \quad 1.00 \quad 1.08 \quad 1.13 \quad 1.21 \quad 1.23 \quad 1.12 \quad 1.21 \quad 1.27 \quad 1.36 \quad 1.46 \quad 1.49$

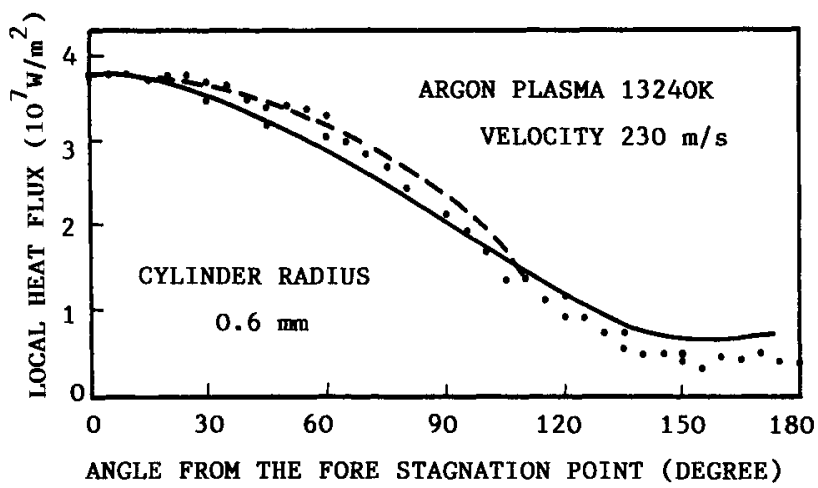

Fig. 3. Comparison of the computational local heat fluxes with experimental data for a cylinder immersed in an argon plasma flow (plasma temperature $13240 \mathrm{~K}$, velocity $230 \mathrm{~m} / \mathrm{s}$, cylinder diameter $1.2 \mathrm{~mm}$; full line - iwo-dimensional computation; dotted line -- boundary layer computation; experimental data is taken from ref. [43]).

However, the experimental heat flux obtained by using the dynamic thermocouple method for argon plasma (temperature $13240 \mathrm{~K}$, velocity $230 \mathrm{~m} / \mathrm{s}$, and sphere diameter $0.86 \mathrm{~mm}$ ) in ref. [17] is less than the predictions by Eqs. (1) $2-(8)$, and much lower than that predicted by Eq. (8) - The experimental heat flux $\left(12.6 \mathrm{~W} / \mathrm{mm}^{2}\right)$ is even lower than that corresponding to the case of pure heat conduction ( $\mathrm{Nu}_{\mathrm{s}}=2$ ) if LTE plasma properties are employed. On the other hand, agreement between the experimental data of the local heat flux to a cylindrical tube [ref.43] and corresponding computational values based on the LTE argon properties is very good, as shown in Fig. 3. This contradiction also exiscs when we compared experimental heat fluxes obtained in our Lab using the dynamic thermocouple method and a water-cooling cylindrical tube with corresponding computational values based on LTE plasma properties. Namely, computation agrees well with experiment for the cylinder case, but disagreement is found for the sphere case. It is not clarifled that whether the dynamic thermocouple method itself is responsible to this puzzling phenomenon. Unfortunately, so far no reliable experimental data are available for small particles with sizes typical for thermal plasma processing. Experimental work of this kind is highly desirable.

\section{Free-molecule regime}

As gas pressures are low or/and when particle diameters are small so that the mean free path length of gas particles is much greater than the particle diameter, heat transfer would be within the free - molecule regime. A plasma - particle heat transfer expression is needed for the modeling work under such conditions. Even for the cases with moderate Knudsen numbers (transition regime), the analytical expression for plasma-paricle heat transfer for the extreme case of free - molecule flow (FMF) regime is still required if an interpolation formula is employed to calculate the plasma-particle heat transfer in the transition regime [ref. 14]. As an extension of the kinetic theory treatment for FMF heat transfer under ordinary temperatures and of ref. [22], refs. [34 \& 35] gave plasma-particle heat transfer expressions for FMF regime with taking both convection and gas ionization into account. 
Main assumptions employed in refs. [34 \& 35] include: 1) The Knudsen number is so great that plasma-particle heat transfer is within the FMF regime; 2) Thin plasma sheath thickness; 3) Maxwellian velocity distribution function is applicable to each gas species (atoms, ions and electrons); 4) Ions recombine with electrons at the particle surface accompanied by the release of the ionization energy; 5) Completely diffuse reflection of gas particles at the sphere surface. For a metallic sphere, closed analytical expressions have been obtained [ refs. 34]. Components due to, respectively, atoms, ions and electrons in the total heat flux to a metallic sphere with diameter d are as follows [ref. 34]:

$$
\begin{aligned}
& Q_{a}=\left(\pi d^{2}\right)\left(\frac{1}{8}\right) a_{a} n_{a} \bar{v}_{a} K T_{h}\left\{\left(s_{h}^{2}+2.5-2 T_{w} / T_{h}\right) \exp \left(-s_{h}^{2}\right)\right. \\
& \left.+\frac{\sqrt{\pi}}{s_{h}}\left[s_{h}^{4}+3 s_{h}^{2}+0.75-\left(T_{w} / T_{h}\right)\left(1+2 s_{h}^{2}\right)\right] \operatorname{erf}\left(s_{h}\right)\right\} \\
& Q_{i}=\left(\pi d^{2}\right)\left(\frac{1}{8}\right) a_{i} n_{i} \bar{v}_{i} K T_{h}\left\{\left[\sqrt{\pi} \frac{1}{2 s_{h}}+s_{h}\right) \operatorname{erf}\left(s_{h}\right)+\exp \left(-s_{h}^{2}\right)\right]\left[s_{h}^{2}+2.5\right. \\
& \left.\left.\quad-2 T_{w} / T_{h}+(E-W) /\left(a_{i} K_{h}\right)+\operatorname{eV} /\left(K_{h}\right)\right]-\left[\sqrt{\pi} /\left(2 s_{h}\right)\right] \operatorname{erf}\left(s_{h}\right)\right\} \\
& Q_{e}=\left(\pi d^{2}\right)\left(\frac{1}{4}\right) n_{e} \bar{v}_{e}\left(2 K T_{e}+W\right) \exp \left[-\operatorname{eV} /\left(K T_{e}\right)\right]
\end{aligned}
$$

Where $n, \bar{v}, T$ and $s$ are number density of gas particles, average thermal motion speed of gas particles, temperature and the speed ratio defined as $s=u /\left(2 \mathrm{KT}_{i} / \mathrm{m}_{4}\right)^{1 / 2} ; \mathrm{e}, \mathrm{K}, \mathrm{E}, \mathrm{W}$ and $\mathrm{V}$ are elementary charge quantity, Boltzmann constant, gas ionlzation energy, work function of sphere material and the absolute value of the floating potential on the sphere: "a" is the thermal accommodation coefficient; and subscripts $a, i, e, h$ and $w$ denote atoms, ions, electrons, heavy-particles (atoms and ions) and sphere wall, respectively.

Total heat flux to a metallic particle due to all three gas species is

$$
Q=Q_{a}+Q_{i}+Q_{e}
$$

which is directly proportional to the square of the particle diameter. It has been shown that the total heat fluxes are almost the same for metallic or nonmetallic spheres, although there exists appreciable difference between these spheres in the distributions of the local heat flux and of the floating potential on the sphere surface [refs. 34 \& 35]. Heat transfer is mainly caused by atoms at low plasma temperatures (e.g. less than $7000 \mathrm{~K}$ for argon plasma), while it can be dominantly attributed to lons and electrons at high plasma temperatures wich appreciable gas ionization [refs. 34 \& 35]. Ref. [35] studied the effects on plasma-particle heat transfer within the FMF regime of the gas pressure and of the heavy-particle/electron temperature ratio. Total heat flux increases approximately linearly with the increase of the gas pressure, ano reduces as the heavy-particle/electron temperature ratio, $T_{\text {, }} / \mathrm{T}_{\text {, ciecreases. }}$ for a given electron temperature. Ref. [46] showed that $T_{b} / T$ can be much less than 1.0 for a high temperature gas flow after supersonic expansion. This two-cemperature effect may be important for the modeling of low-pressure plasma spraying.

Unfortunately, so far no direct experimental daca are available to check the predicted results of Eqs. (9) - (12). It is extremely important to know whether the kinetic theory treatment which has been successfully employed under ordinary temperature fuF conditions, can be extended to the conditions of thermal plasma FlF since many additional assumptions have been used, as mentioned above. Hence, an analysis is performed recently concerning heat transfer from a rarefied olasma flow to a metallic wire based on the same assumptions as for the sphere because some experimental data can be obtained from ref. [48]. The specific heat fuxes, after being circumferentially averaged, due to, respectively, atoms, ions and electrons are derived co be as follows:

$$
\begin{aligned}
& q_{a}=\left(\frac{1}{4}\right) a_{a} n_{a} \bar{v}_{a} K T_{h} \exp \left[-s_{h}^{2} / 2\right]\left\{\left(2+s_{h}^{2}-2 T_{w} / T_{h}\right) I_{0}\left(s_{h}^{2} / 2\right)\right. \\
&\left.+\left(2.5+s_{h}^{2}-2 T_{w} / T_{h}\right) s_{h}^{2}\left[I_{0}\left(s_{h}^{2} / 2\right)+I_{1}\left(s_{h}^{2} / 2\right)\right]\right\} \\
& q_{i}=\left(\frac{1}{4}\right) a_{i} n_{i} \bar{v}_{i} K T_{h} \exp \left[-s_{h}^{2} / 2\right]\left\{\left[2+s_{h}^{2}-2 T_{w} / T_{h}+(E-W) /\left(a_{i} K T_{h}\right)\right.\right. \\
&\left.+e v_{0} /\left(K T_{h}\right)\right] I_{0}\left(s_{h}^{2} / 2\right)+\left[2.5+s_{h}^{2}-2 T_{w} / T_{h}+(E-W) /\left(a_{i} K_{h}\right)\right. \\
&\left.\left.+e v_{0} /\left(K T_{h}\right)\right] s_{h}^{2}\left[I_{0}\left(s_{h}^{2} / 2\right)+I_{1}\left(s_{h}^{2} / 2\right)\right]\right\} \\
& q_{e}=\left(\frac{1}{4}\right) n_{e} \bar{v}_{e}\left(2 K T_{e}+W\right) \exp \left[-e v_{o} /\left(K T_{e}\right)\right]
\end{aligned}
$$


and the resultant heat flux due to all the three species is

$$
q=q_{a}+q_{i}+q_{e}
$$

where $V_{0}$ is the absolute value of the applied voltage between the metallic wire and the plasma (anode of the plasma-jet generator). I and $I_{1}$ are the zero-order and the first-order Bessel functions.

The experimental data in ref. [48] are obtained by sweeping a tungsten wire with diameter of $0.15 \mathrm{~mm}$ across a rarefied plasma jet with parameters of gas pressure 0.005 atm, temperature about $9000 \mathrm{~K}$ and flow velocity about $1300 \mathrm{~m} / \mathrm{s}$ (or s is about 0.6 ). Variarion of the experimental heat flux with the applied voltage is shown in Fig. 4 and compared with the predicted results of Eqs. (13) - (16). It is seen that the predicted heat fluxes by Eqs. (13) - (16) agree well with the experimental data in the magnituce of the heat flux and in the slope of the heat flux variation with the applied voltage if one takes the plasma temperature $\mathrm{T}_{\mathrm{h}}=\mathrm{T}_{\mathrm{e}}$ $=8400 \mathrm{~K}$, which is only slightly lower than the measured value in ref. $[48] \mathrm{T}_{\mathrm{h}}=\mathrm{T}_{\mathrm{e}}=9000 \mathrm{~K}$.

Such a good agreement between the experimental data and the theoretical predicíons of Eqs. (13) - (16) provides a desirable support for the kinetic theory treatment under thermal plasma conditions, on which Eqs. (9) - (12) are based. However, further direct comparison between the theoretical results of Eqs. (9) - (12) and FMF plasma-particle heat transfer experiments is needed, especially for the case with smaller particle sizes typical for the thermal plasma processing.

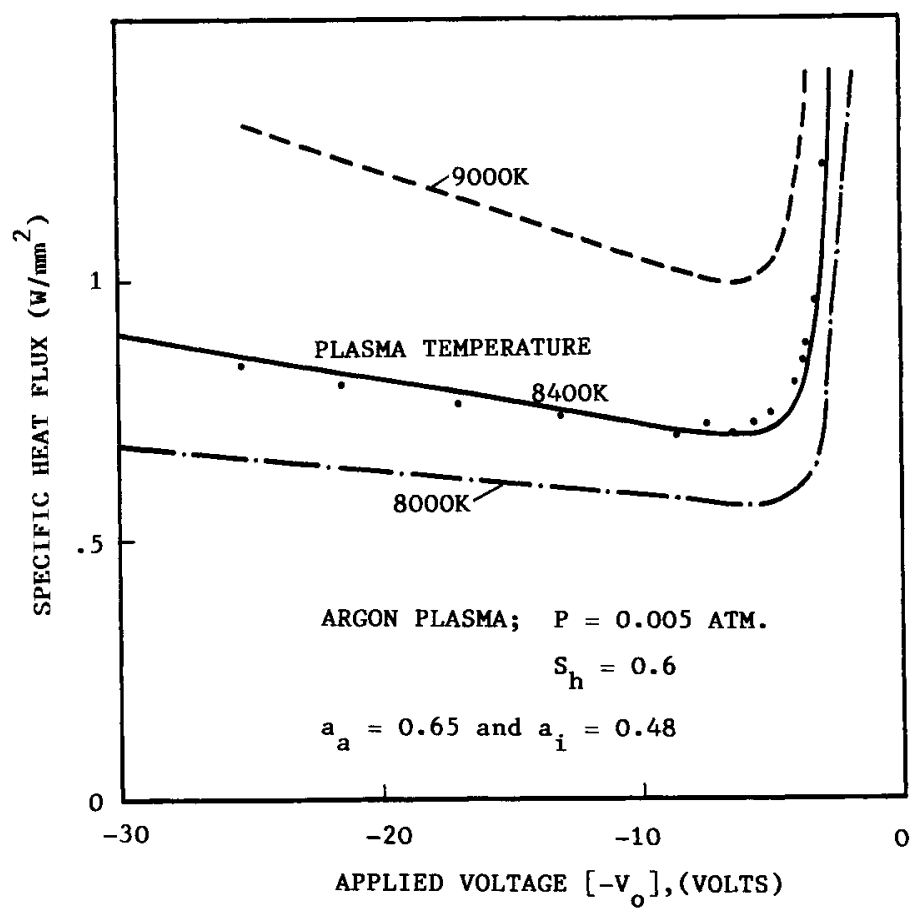

Fig. 4. Comparison of the predicted heat $f 1 u x$ of Eqs. (13) - (16) with experimental data [ref. 48] for a cylindrical wire exposed to a rarefied argon plasma flow ́pressure, 0.005 atm; temperature, about 9000K ; velocity, about 1300 $\mathrm{m} / \mathrm{s}$; 1 ines, predictions ; dots, experimental data from ref. [48]).

\section{Transition regime $\left(10^{-3}<\mathrm{Kn}<10\right)$}

Today there is no strict analysis covering the whole transition regime under thermal plasma conditions, and no systematic experimental study has been conducted.

For the case with small knudsen numbers within the temperature jump regime, an analysis based on the so-called heat-conduction-potential jump approach was presented concerning the Knudsen effect on particle heat transfer under thermal plasma conditions with or without convection [refs. 25 \& 27]. This analysis has been employed to study the effect of gas pressure on plasma-particle heat transfer under soft vacuum conditions, and to explain why the heat flux from the thermal plasma to the particle in question reduces under soft vacuum 
plasma spraying conditions [ref. 47]. This analysis has also been extended co study the effect of the Knudsen number on mass transfer between a single particle and a thermal plasma [refs. 31 \& 32]. An interesting finding which is different from the plasma-particle heat transfer case is that the Knudsen effect may reduce but also may enhance the masss transfer between the thermal plasma and the particle.

However, including the contribution due to ionized species (ions and electrons) to the heat transfer within the Knudsen layer, ref. [41] obtained another analytical result which predicted a quite different trend from the original analysis [ref. 25] concerning the variation of the plasma-particle heat flux with the particle diameter within the jump regime. The analysis in ref. [41] neglected contribution due to the presence of temperature gradient at the outer edge of the Knudsen layer to the plasma-particle heat flux, and it was based on a thick plasma sheath assumption. The thick sheath assumption is less realistic because ionized species contribute an appreciable fraction to the total heat flux only at high plasina cemperature associated with very chin plasma sheath [ref. 34].

Assuming a thin plasma sheath and considering the contribution to heat transfer within the Knudsen layer due to the presence of temperature gradient at the outer edge of the knudsen layer, the following heat transfer relation can be obtained:

$$
\left[\frac{Q}{a}\right]=\left(\pi d^{2}\right)\left[\left(N_{a}+N_{i}+N_{e}\right) 2 K\left(T *-T_{W}\right)+N_{i}\left(E+e V+2 K T_{w}\right)\right]+\left(\frac{d}{d+2 z}\right)\left[\frac{Q}{2}\right]
$$

where $N$ and $z$ are the gas paricle flux [ref. 34] and the jurnj discance or the Knudsen layer thickness [ref. 25]; $\mathrm{T}^{*}$ is the gas temperature at the outer edge of the Knudsen layer; and $Q$ is the total heat flur to the sperical particle with diameter d.

The right hand side of Eq. (17) consists two terms : the first denotes heat transfer due to atoms, ions and elctrons within the Knudsen layer with thickness $z$ itreated as FMF heat transfer [ref. 34]) for the case of complete thermal accommodation, and the second is the conductive heat flux inward to the particle surface due to the presence of temperature gradient at the outer edge of the Knudsen layer.

As the jump distance $z$ is small in comparison with the particle diameter (or small Knudsen number), the jumped gas temperature $T^{*}$ does not deviate from particle temperature $T_{w}$ too much and thus gas ionization within the Knudsen layer is negligible. For this situation, Eq. (17) would reduce to the following form:

$$
\left[\left(\frac{2-a}{2 a}\right) q\right] \text { or }\left[\left(\frac{2-a}{2 a}\right)\left(\frac{d S}{d r}\right)_{w}\right]=\left(m_{a} N_{a}\right)\left(\frac{1+\gamma}{2 \gamma}\right)(\bar{C} p / \bar{k})\left(S *-s_{w}\right)
$$

where we have employed the relations

and

$$
\left(T *-T_{W}\right)=\left(S^{*}-S_{w}\right) / \bar{k}
$$

$$
2 K\left(T *-T_{W}\right)=\left[\left(\frac{1+\nu}{2 \gamma}\right)\right]\left(h *-h_{W}\right)=\left(\frac{1+\gamma}{2 \gamma}\right) \bar{C}_{p}\left(T *-T_{W}\right)
$$

From the assumed relation [ref.25]

$$
S^{*}-S_{w}=z\left(\frac{d S}{d r}\right)_{W}
$$

the jump distance is obtained as

$$
\left.z=\left(\frac{2-a}{a}\right)\left(\frac{\gamma}{1+\gamma}\right)\left[\frac{1}{\left(m_{a} N_{a}\right.}\right)(\bar{k} / \bar{C} p)\right]
$$

and the heat flux ratio with to without accounting for the Knudsen effect, $Q / Q_{c}$, is [ref.25]

$$
\mathrm{Q} / \mathrm{Q}_{\mathrm{C}}=1 /\left[1+\left(\mathrm{z} / \mathrm{r}_{\mathrm{w}}\right)\right]
$$

where $m$ and $\gamma$ are atom mass and the specific heat ratio (monatomic gas is assumed in the derivation); $T *, h *$ and $S *$ are jumped temperature or the gas temperature at the outer edge of the knudsen layer, gas specific enthalpy and heat-conduction-potential corresponding to the temperature; $\bar{k}$ and $\bar{c}_{p}$ are the integrally averaged thermal conductivity and specific heat from particle wall temperature $T_{w}$ to the jumped temperature $T^{*}$ [ref. 25]; $Q_{C}$, continuum case

If let the mass flux of gas particles within the Knudsen layer, $\left(\mathrm{m}_{\mathrm{a}} \mathrm{N}_{\mathrm{a}}\right)$, is equal to $\boldsymbol{P}_{\mathrm{w}} \overline{\mathrm{v}}_{\mathrm{w}} / 4$, the same results as in ref. [25] would be obtained. However, a more suitable expression for the mass flux of gas particles within the knudsen layer is [ref. 49]

$$
\left(\mathrm{m}_{\mathrm{a}} \mathrm{N}_{\mathrm{a}}\right)=\left[\left(\frac{1}{4}\right) \rho_{\mathrm{w}} \overline{\mathrm{v}}_{\mathrm{w}}\right]\left[\frac{2}{1+\left(\mathrm{T} * / \mathrm{T}_{\mathrm{W}}\right)^{1 / 2}}\right]
$$


Since this modification [ref. 49] gives less mass flux of gas particles within the Knudsen layer in comparison with ref. [25], more pronounced Knudsen effect on plasma-particle heat transfer would be expected.

Gas ionization has been completely ignored in deriving Eqs. (18) - (21), namely $\mathbb{N}_{i}$ and $\mathbb{N}_{e}$ has been assumed to be zero. On the other hand, as the jump distance $z$ is still small bui T* is high so that gas ionization can not be neglected, similar but somewhat complicated calculation can be conducted by using Eq. (17). Some typical calculaced results obtained from this improved analysis are shown in Fig. 5 and compared with the previous results [refs. $25 \& 41]$. It is seen from Fig. 5 that the same variation with the particle diameter of the predicted heat flux as in ref. [25] is obtained, although more pronounced knudsen effect is observed. As mentioned above, this difference is partially caused by using here a less mass flux of gas particles as Eq. (21) than in ref. [25].

It is also obvious from Eq. (17) that the second term on the right hand would be negligible and the jumped cemperature $T *$ would approach the plasma temperature $T$ as great Knudsen number (e.g. Kn greater than 10) or great jump distance (z much greater chan particle diamecer a) is concerned. Almost the same result as that for the fíf regime mentioned above would be obcained except that an average thermal accommodation coefficient "a" has been used here and the electron cemperature has been assumed to be equal to the heavy-particle temperature.

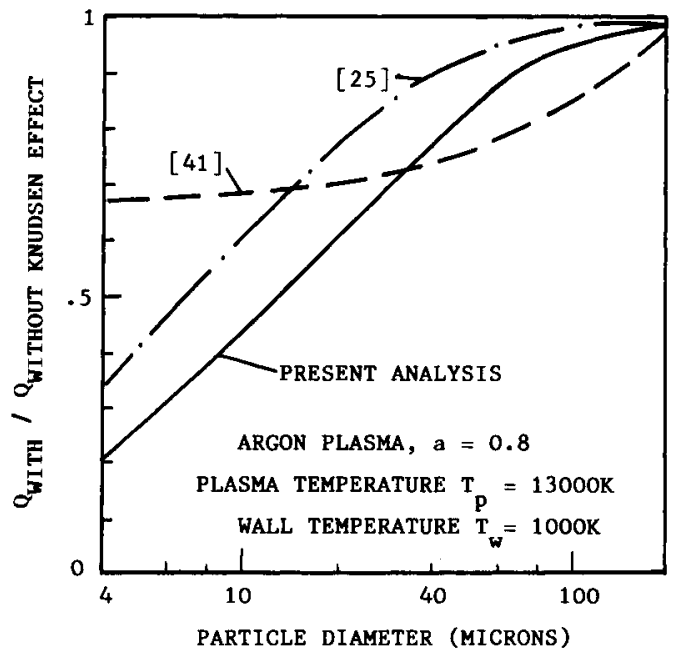

Fig. 5. Comparison of the predicted heat flux ratio with to without accounting for the Knudsen effect (jump regime; plasma cemperature $13000 \mathrm{~K}$, wall cemperature 1000K; [25], withouc ionization effect; [41], thick plasma sheath; the present analysis, thin plasma sheath).

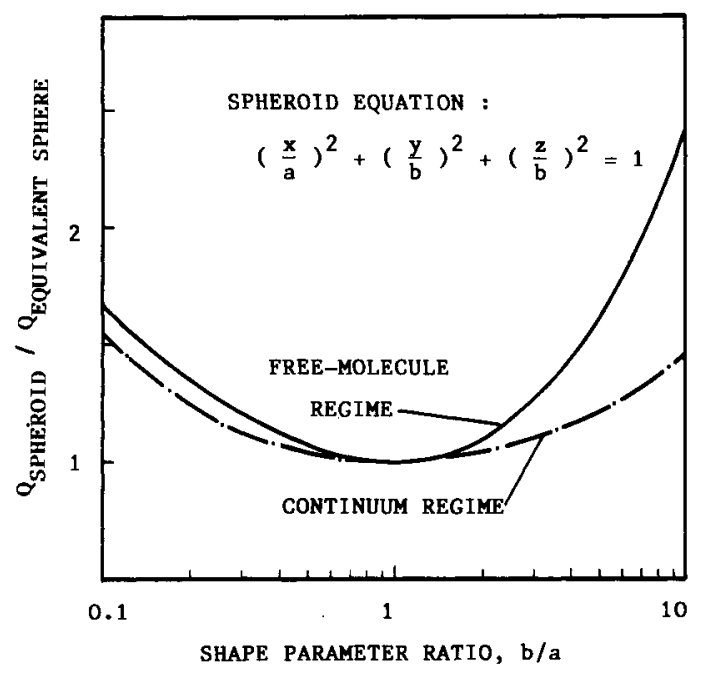

Fig. 6. Effect of particle non-sphericity on heat iransfer to a parícle for both coninuum and free-nolecule regimes ( $a$ and $b$ are paramerers in the spheroid equation $\left.\left(\frac{x}{a}\right)^{2}+\left(\frac{y}{b}\right)^{2}+\left(\frac{z}{b}\right)=1\right)$.

\section{SOME OTHER COMPLICATED FACTORS}

Experimental daca of ref. [50] show that the evaporation behavior of a solute particle in the flame spectroscopy assumes abrupt change in its evaporation process. For a large partcle with ciameter of micions, variation with time of the instantaneous diameter of the solute particle sacisfies

$$
d^{2}=c_{01}^{2}-k_{1} \tau
$$


gas for the continum regime. However, after the particle diameter is less than a critical value, its evaporation behavior changes to

$$
d=d_{02}-k_{2} t
$$

which is the typical evaporation behavior for the FMF regime. Where $t$ is the time; $d_{01}$ and $\mathrm{d}_{02}$ are the initial particle diameters for both situations; $k_{1}$ and $k_{2}$ are experimental constants, repectively. Up to now, no satisfactory explanation is available for the abrupt change in the observed particle evaporation behavior [ref. 51], since the existing theory predicts a continuous change of the particle evaporation behavior within a much wider range of solute particle diameter from the continuum regime to the FMF regime (Kn varies from $10^{-3}$ to 10). Although the observed daca in ref. [50] were obtained in the flame acomic spectroscopy in which lower gas temperatures (about 2500K) are involved, similar evaporation behavior is expected to be observed under plasma conditions (ICP atomic spectroscopy). An inceresting finding which is probably related to this puzzling phenomenon was obtained in ref. [52], which showed that a particle with low thermal conductivity may explode during intense heating due to the development of great thermal stress within the particle.

Particle shape may be non-spherical. In addition, a moving droplet would deviate more or less from the spherical shape due to the nonuniform action of external forces. The effect of particle shape parameter on heat transfer to a rotational spheroid can be readily escimated for the extreme cases of continuum and FMF regimes and for the case of pure heat conduciion. For the extreme case of FMF regime, since the local heat flux is independent of the angle position on parcicle surface as $R e=0$, the heat flux ratio of the spheroid to its equivaleni sphere with the same volume is equal to their surface-area ratio, which is easily calculated by using a mathematical handbook [ref. 53]. On the other hand, for the continuum regime, the local heat flux is not uniform over the particle surface so the heat flux ratio is not equal to the surface-area ratio. However, heat transfer to a spheroid immersed in an infinite stagnant continum plasma can be obtained by using a method analogous to that used in static electricity [ref. 54], so the heat flux ratio of the spheroid to its equivalent sphere can be obtained. Calculated results for both continuum and FMF regimes are shown in Fig. 6 . It is seen from Fig. 6 that if the particle deformation is not too serious (shape parameters a is approximately equal to $b$ ), the total heat flux to a spheroid is only slightly higher than that to its equivalent sphere with the same volume. Shape effect for the continuum regime is less than that for the Filf regitne.

So far one-Gimensional unsteady heat conduction equation is solved to predict the particle heating history in the modeling work. This approach is somewhat too simplified for a particle with low thermal conductivicy since highly non-uniform heat flux distribution over the particle surface would cause non-uniform heating under flowing plasma conditions. In addition, if any chemical reactions exist on the particle surface or within the particle, the study of plasma-particle heat transfer would be further complicated. It is obvious that more investigations are required to clarify effects of these complicated factors on heat iransfer.

\section{CONCLUSIONS}

Although a great progress has been achieved recently in the plasma-particle heac transfer study, much nore work renains to be conducted. Particularly, reliable exierimental daca aze highly desirable concerning heat transfer to small particles (microns to tens of microns) to check the available heat transfer expressions and/or to clarify the effects of various complicated factors including those within the thermal boundary layer anc on jarticle surface.

\section{Acknowledgement}

This work was supported by the National Natural Science Founciation of China.

\section{REFERENCES}

1. P. Fauchais et al., Pure and appliec chenistry 57, 1171-1178 (1935).

2. K. Akashi, Pure and Applied Chemiscry 57, 1197-1206 (1985).

3. 1... Boulos, Pure anc Appliec Chenistiy 57, 1321-1352 (1985).

4. H.J. Zarvey and T.if. Veyer, iecallurgical Transactions 9B, 515-521 (1978).

5. B. Gal-Or, ASTE J. Eng. Eor Power 102, 589-593 (1930).

6. Xi Chen and Bangchu Lin, ISPC7 3 ; \$86-891 (1985).

7. T. Yoshica and K. Akashi, J. Appl. Phys. 48, 2252-2260 (1977).

8. 11.I. Boulos, IEEE Transactions on Plasma Science $6,93-106$ (1978).

9. J.K. Fiszdon, Int. . . .eat Mass Transfer 22 , 749-761 (1979).

10. Y.C. Lee, K.C. hisu and E. Pfenceis, ISPC5 $\frac{2}{2}, 795-803$ (1979). 
11. A. Varcielle, K. Vardelle and P. Fauchais, Plasma Chem. Plasma Proces. 2, 255-291 (1982). 12. M. Vardelle, A. Vardelle, P. Fauchais and $\overline{\text { M. }}$. Boulos, AIChE J. 29, $23 \overline{6}-291$ (1983). 13. Xi Chen, Y.C. Lee and E. Pfencier, ISPCE $1,51-58$ (1983).

14. D. Apelian, D. Wei ana . Paliwal, Thin Solid Films 118, 395-407 (1984).

15. N. El-Kaddah, Z. I1ckelliget and $Z$. Szekely, Métallurgical Trans, 15B, 59-70 (1984).

16. P. Proulx, ¿. Mostaghimi anć M. I. Boulos, int. I. heat Mass Transfer 28, 1327-35 (1985).

17. A. Kanzawa, Heat Transfer--Lapanese Research 2, 63-70 (1973).

18. ¿.A. Lewis and W. H. Gauvin, AIChE J. 19, 982-990 (1973).

19. i.V. Kalganova and V.S. Klubnikin, Figh Temperature 14, 408-409 (1976).

20. I.s. Burov, Phys. Chem. of iiaterial Processing ilo.4, 42-49 (1979; in Russian).

21. N.N. Sayegh and W.K. Gauvin, AIChE J. 25, 522-534 (1979).

22. N.N. Rykalin et al., High Temperature $\overline{19}, 404-411$ (1981).

23. Xi Chen and E. Pfender, Plasma Chem. PIasma Proces. 2, 185-212 (1982).

24. Xi Chen and E. Pfender, Plasma Chem. Plasma Proces. $\frac{2}{2}, 291-314$ (1982).

25. Xi Chen and E. Pfender, P1asma Chem. Plasma Proces. 3 , 97-113 (1983).

26. E. Bourdin, P. Fauchais and M.I. Boulos, Int. J. Heat Mass Transfer 26, 567-582 (1983).

27. Xi Chen and E. Pfender, Plasma Chem. Plasma Proces. 3, 351-366 (1983).

28. Xi Chen, Y.P. Chyou, Y.C. Lee and E. Pfender, Plasma Chem. Plasma Proces. 5, 119-141 (1985).

29. Xi Chen, in Heat Transfer Science and Technology (ed. by B.X. Wang) 748-755, hemisphere, Washington (1987).

30. I. Groma and B. Veto, Int. J. Heat Mass Transfer 29, 549-554 (1986).

31. S.V. Joshi et al., Plasma Chem. Plasma Proces. 6, 281-298 (1986).

32. S.V. Joshi et al., Int. J. Keat Mass Transfer $2 \overline{9}, 1565-1573$ (1986).

33. Xi Chen, Proceedings of the International Conference on Plasma Science and Technology (ed. by C.H. Wu et al.), 107-114, Science Press, Beijing (1986).

34. $\mathrm{Xi}$ Chen and Ping He, heat Transfer--1986 6, 3067-3072, hemisphere, Washington (1986).

35. Xi Chen and Ping He, Plasma Chem. Plasma Proces. 6, 313-333 (1986).

36. S.V. Dresvin et al., Izv. SO Ali SSSR, Ser. Tech. Ści. Vyp. 1, 69-77 (1986).

37. B. Waldie, The Chemical Engineer May issue, 188-193 (1972).

38. E. Pfender, Pure and Applied Chemistry 57, 1179-1198 (1985).

39. Y.C. Lee, Ph. D. Thesis, University of Minnesota (1984).

40. E. Pfender and Y.C. Lee, Plasma Chem. Plasma Proces. 5, 211-237 (1985).

41. Y.C. Lee, Y.P. Chyou and E. Pfender, Plasma Chem. Plasma Proces. 5, $391-414$ (1985).

42. P. Menbus and M. Elayoubi, Can. J. Phys. 61, 683-690 (1983).

43. A. Kanzawa and S. Nonouchi, Int. Chem. Eng 16, 184-189 (1970).

44. M. Omiya and A. Kanzawa, heat Transfer -- Japanese Research 6, 81-83 (1977).

45. E. Pfender and Y.C. Lee, in laterial Res. Soc. Symp. 30, 141-150, North-Holland, New York (1984).

46. F.I. Zakharov et al., J. of Eng. Phys. 44, 620-632 (1983; in Russian).

47. Xi Chen and E. Pfender, ASME J. Eng. for Gas Turbines and Power 107, 147-151 (1985).

48. T. Honda, T. Hayashi and A. Kanzawa, Int. J. Heat Mass Transfer $24,1247-1255$ (1981).

49. Y. Wu, J. Chem. Phys. 52, 1494-1498 (1970).

50. Y. Pak anc G.M. Hieftje, Spectrochimica 40B, 209-216 (1985).

51. G.M. Hieftje, Private communication (1987).

52. Minglun Xue, Hechanics and Practice 8 (2), 31-33 (1986; in Chinese).

53. G.A. Korn and T. M. Korn, Mathematical Hancbook for Scientists and Engineers, 883, McGraw-Hill, New York, 2nd edition (1968).

54. E. Weber, Electromagnetic Fields Theory and Applications, Vol.1--îaping of Fields, 446-453, Wiley, New York (1950). 